\begin{tabular}{|c|c|c|}
\hline & Global Journal of Mathematical Analysis, 5 (2) (2017) 37-42 & \\
\hline & Global Journal of Mathematical Analysis & \\
\hline SPC & $\begin{array}{c}\text { Website: www.sciencepubco.com/index.php/GJMA } \\
\text { doi: } 10.14419 / \text { gima.v5i2. } 7703 \\
\text { Research paper }\end{array}$ & \\
\hline
\end{tabular}

\title{
On Properties of meromorphic solutions of difference Painlevé I and II equation
}

\author{
Wei Min Xue ${ }^{1 *}$ and Yan Mei Teng ${ }^{2}$ \\ ${ }^{1}$ School of Mathematics and Systems Science, Beihang University, Beijing, P. R. China \\ ${ }^{2}$ School of Mathematics and Systems Science, Beihang University, Beijing, P. R. China \\ *Corresponding author E-mail: xwm_2017@163.com
}

\begin{abstract}
In this paper, we investigate some properties of finite order transcendental meromorphic solutions of difference Painlevé I and II equations, and obtain precise estimations of exponents of convergence of poles of difference $\Delta w(z)=w(z+1)-w(z)$ and divided difference $\frac{\Delta w(z)}{w(z)}$, and of fixed points of $w(z+\eta)(\eta \in \mathbb{C} \backslash\{0\})$.
\end{abstract}

Keywords: Difference; Divided Difference; Difference Painlevé Equations; Meromorphic Function

\section{Introduction and Results}

In this paper, we assume that the reader is familiar with the basic Nevanlinna's value distribution theory of meromorphic functions (see[2,10]). In addition, we use the notation $\sigma(f)$ to denote the order of growth of the meromorphic function $f(z), \lambda(f)$ and $\lambda\left(\frac{1}{f}\right)$ to denote, respectively, the exponent of convergence of zeros and poles of $f(z)$. We also use the notation $\tau(f)$ to denote the exponent of convergence of fixed points of $f(z)$ which is defined as

$$
\tau(f)=\limsup _{r \rightarrow \infty} \frac{\log N\left(r, \frac{1}{f(z)-z}\right)}{\log r} .
$$

We denote by $S(r, f)$ any quantify satisfying $S(r, f)=o(T(r, f))$, as $r \rightarrow \infty$, possibly outside a set with finite measure.

Recently, a number of papers (including $[3-9,11-14]$ ) have focused on complex difference equations and difference analogues of Nevanlinna's theory. As the difference analogues of Nevanlinna's theory are investigated $[8,12]$, many results on the complex difference equations are rapidly obtained.

Halburd and Korhonen [9] used value distribution theory and a reasoning related to the singularity confinement to single out the difference Painlevé I and II equations from difference equation

$$
w(z+1)+w(z-1)=R(z, w),
$$

where $\mathrm{R}$ is rational in both of its arguments. They proved that if (1.1) has an admissible meromorphic solutions of finite order, then either $w$ satisfies a difference Riccati equation, or (1.1) may be transformed into some classical difference equations, which include difference Painlevé I equations

$$
w(z+1)+w(z-1)=\frac{a z+b}{w(z)}+c,
$$

$$
\begin{gathered}
w(z+1)+w(z-1)=\frac{a z+b}{w(z)}+\frac{c}{w^{2}(z)}, \\
w(z+1)+w(z)+w(z-1)=\frac{a z+b}{w(z)}+c,
\end{gathered}
$$

and difference Painlevé II equation

$$
w(z+1)+w(z-1)=\frac{(a z+b) w(z)+c}{1-w^{2}(z)},
$$

where $a, b$ and $c$ are constants.

In 2010, Chen and Shon [13] investigated some properties of meromorphic solutions of difference Painlevé I equation (1.2) and difference Painlevé II equation (1.5), and proved the following results.

Theorem B. (See [13]) Let $a, b, c$ be constants with $a c \neq 0$. If $w(z)$ is a finite-order transcendental meromorphic solution of the difference Painlevé II equation (1.5), then:

(i) $w(z)$ has at most one non-zero finite Borel exceptional value;

(ii) $\lambda\left(\frac{1}{w}\right)=\lambda(w)=\sigma(w)$;

(iii) $w(z)$ has infinitely many fixed points and satisfies $\tau(w)=\sigma(w)$. Theorem C. (See [13]) Let $a, b, c$ be constants with $a \neq 0$. If $w(z)$ is a finite-order transcendental meromorphic solution of the difference Painlevé I equation (1.2), then

(i) $w(z)$ has at most one non-zero finite Borel exceptional value;

(ii) $\lambda\left(\frac{1}{w}\right)=\lambda(w)=\sigma(w)$;

(iii) $w(z)$ has infinitely many fixed points and satisfies $\tau(w)=\sigma(w)$. In 2011, Chen [14] investigated some properties of meromorphic solutions of difference Painlevé I equation (1.3) and obtained the following result.

Theorem D. (See [14]) Let $a, b, c$ be constants such that $a c \neq 0$. Suppose that $w(z)$ is a finite-order transcendental meromorphic solution of the difference Painlevé I equation (1.3), then

(i) $w(z)$ has no any Borel exceptional value;

(ii) If $p(z)$ is a non-constant polynomial, then $w(z)-p(z)$ has infinitely many zeros and satisfies $\lambda(w-p)=\sigma(w)$. 
In 2012, Chen and Chen [7] investigated some properties of meromorphic solutions of difference Painlevé I equation (1.4) and proved the following result.

Theorem E. (See [7]) Let $a, b, c$ be constants such that $|a|+|b| \neq 0$. If $w(z)$ is a finite-order transcendental meromorphic solution of the difference Painlevé I equation (1.4), then:

(i) $\lambda\left(\frac{1}{w}\right)=\lambda(w)=\sigma(w)$;

(ii) If $p(z)$ is a non-constant polynomial, then $w(z)-p(z)$ has infinitely many zeros and satisfies $\lambda(w-p)=\sigma(w)$.

(iii) If $a \neq 0$, then $w(z)$ has no Borel exceptional value;

If $a=0$, then the Borel exceptional value of $w(z)$ can only come from a set $E=\left\{z \mid 3 z^{2}-c z-b=0\right\}$.

In this paper, we consider some properties of difference and divided difference of transcendental meromorphic solutions of the difference Painlevé I equations (1.2) - (1.4) and Painlevé II equation (1.5), and obtain the following results.

Theorem 1.1. Let $a, b, c$ be constants with $|a|+|b| \neq 0$. If $w(z)$ is a finite-order transcendental meromorphic solution of the difference Painlevé I equation (1.2), then

(i) $\lambda\left(\frac{1}{\Delta w}\right)=\lambda\left(\frac{1}{\frac{\Delta w}{w}}\right)=\sigma(w)$;

(ii) For any $\eta \in \mathbb{C} \backslash\{0\}, \tau(w(z+\eta))=\sigma(w)$.

Theorem 1.2. Let $a, b, c$ be constants with $|a|+|b|+|c| \neq 0$. If $w(z)$ is a finite-order transcendental meromorphic solution of the difference Painlevé I equation (1.3), then

(i) $\lambda\left(\frac{1}{\Delta w}\right)=\lambda\left(\frac{1}{\frac{\Delta w}{w}}\right)=\sigma(w)$;

(ii) For any $\eta \in \mathbb{C} \backslash\{0\}, \tau(w(z+\eta))=\sigma(w)$.

Theorem 1.3. Let $a, b, c$ be constants with $|a|+|b| \neq 0$. If $w(z)$ is a finite-order transcendental meromorphic solution of the difference Painlevé I equation (1.4), then $\lambda\left(\frac{1}{\Delta w}\right)=\lambda\left(\frac{1}{\frac{\Delta w}{w}}\right)=\sigma(w)$.

Theorem 1.4. Let $a, b, c$ be constants with $|a|+|b|+|c| \neq 0$. If $w(z)$ is a finite-order transcendental meromorphic solution of the difference Painlevé II equation (1.5), then

(i) $\lambda\left(\frac{1}{\Delta w}\right)=\lambda\left(\frac{1}{\frac{\Delta w}{w}}\right)=\sigma(w)$;

(ii) For any $\eta \in \mathbb{C} \backslash\{0\}, \tau(w(z+\eta))=\sigma(w)$.

Remark 1.1. From the proofs of Theorems 1.1-1.4, we can also obtain that $\lambda\left(\frac{1}{w}\right)=\sigma(w)$ and $\sigma\left(\frac{\Delta w}{w}\right)=\sigma(\Delta w)=\sigma(w)$.

Remark 1.2. Generally, $\tau(w(z+\eta)) \neq \tau(w(z))$, where $\eta \in$ $\mathbb{C} \backslash\{0\}$. For example, $w(z)=e^{z}+z, w(z+1)=e e^{z}+z+1, w(z)$ has no any fixed points and $\tau(w(z))=0$, but $w(z+1)$ has infinitely many fixed points and satisfies $\tau(w(z+1))=\sigma(w(z))=1$.

Example 1.1. The meromorphic function $w(z)=\frac{e^{i \pi z}-1}{e^{i \pi z}+1}$ satisfies the difference Painlevé I equation

$$
w(z+1)+w(z-1)=\frac{2}{w(z)},
$$

with $a=c=0, b=2$ satisfying $|a|+|b|=2(\neq 0)$. We see that

$$
\begin{gathered}
\Delta w(z)=\frac{e^{i \pi(z+1)}-1}{e^{i \pi(z+1)}+1}-\frac{e^{i \pi z}-1}{e^{i \pi z}+1}=\frac{4 e^{i \pi z}}{e^{i 2 \pi z}-1}, \\
\frac{\Delta w(z)}{w(z)}=\frac{4 e^{i \pi z}}{e^{i 2 \pi z}-1} \cdot \frac{e^{i \pi z}+1}{e^{i \pi z}-1}=\frac{4 e^{i \pi z}}{\left(e^{i \pi z}-1\right)^{2}}, \\
w(z+\eta)-z=\frac{e^{i \pi(z+\eta)}-1}{e^{i \pi(z+\eta)}+1}-z=\frac{e^{i \pi(z+\eta)}(1-z)-(z+1)}{e^{i \pi(z+\eta)}+1} .
\end{gathered}
$$

Then, $\lambda\left(\frac{1}{\Delta w}\right)=\lambda\left(\frac{1}{\frac{\Delta w}{w}}\right)=\sigma(w)=1, \quad \lambda(\Delta w)=\lambda\left(\frac{\Delta w}{w}\right)=0$. For any $\eta \in \mathbb{C} \backslash\{0\}$, we have $\tau(w(z+\eta))=\sigma(w)=1$.
Example 1.2. The meromorphic function $w(z)=\frac{1}{e^{i 2 \pi z}+z+1}$ satisfies the difference Painlevé II equation

$$
w(z+1)+w(z-1)=\frac{2 w(z)}{1-w^{2}(z)},
$$

with $a=c=0, b=2$ satisfying $|a|+|b|+|c|=2(\neq 0)$. We see that

$$
\begin{aligned}
& \Delta w(z)=\frac{1}{e^{i 2 \pi z}+z+2}-\frac{1}{e^{i 2 \pi z}+z+1}=\frac{-1}{\left(e^{i 2 \pi z}+z+2\right)\left(e^{i 2 \pi z}+z+1\right)} \\
& \frac{\Delta w(z)}{w(z)}=\frac{-1}{\left(e^{i 2 \pi z}+z+2\right)\left(e^{i 2 \pi z}+z+1\right)} \cdot\left(e^{i 2 \pi z}+z+1\right)=\frac{-1}{e^{i 2 \pi z}+z+2}, \\
& w(z+\eta)-z=\frac{1}{e^{i 2 \pi(z+\eta)}+z+\eta+1}-z \\
& =\frac{-z e^{i 2 \pi(z+\eta)}-\left(z^{2}+(\eta+1) z-1\right)}{e^{i 2 \pi(z+\eta)}+z+\eta+1} .
\end{aligned}
$$

Then, $\lambda\left(\frac{1}{\Delta w}\right)=\lambda\left(\frac{1}{\frac{\Delta w}{w}}\right)=\sigma(w)=1, \quad \lambda(\Delta w)=\lambda\left(\frac{\Delta w}{w}\right)=0$. For any $\eta \in \mathbb{C} \backslash\{0\}$, we have $\tau(w(z+\eta))=\sigma(w)=1$.

\section{Some Lemmas}

In order to prove our conclusions, we need the following lemmas.

Lemma 2.1. (See [1], [2, Theorem 2.2.5]) Let $f(z)$ be a meromorphic function. Then for all irreducible rational functions in $f(z)$,

$$
R(z, f(z))=\frac{\sum_{i=0}^{m} a_{i}(z) f(z)^{i}}{\sum_{j=0}^{n} b_{j}(z) f(z)^{j}},
$$

with meromorphic coefficients $a_{i}(z), b_{j}(z)\left(a_{m}(z) b_{n}(z) \not \equiv 0\right)$ being small with respect to $f(z)$, the characteristic function of $R(z, f(z))$ satisfies

$$
T(r, R(z, f(z)))=\max \{m, n\} T(r, f)+S(r, f) .
$$

Lemma 2.2. (See [3, Theorem 2.4], [8]) Let $f$ be a transcendental meromorphic solution of finite order $\sigma$ of the difference equation

$$
P(z, f)=0,
$$

where $P(z, f)$ is a difference polynomial in $f(z)$ and its shifts. If $P(z, a) \not \equiv 0$ for a slowly moving target meromorphic function $a$, that is, $T(r, a)=S(r, f)$, then

$$
m\left(r, \frac{1}{f-a}\right)=O\left(r^{\sigma-1+\varepsilon}\right)+S(r, f),
$$

outside of a possible exceptional set of finite logarithmic measure.

Lemma 2.3. (See [3, Theorem 2.3], [8]) Let $f$ be a transcendental meromorphic solution of finite order $\sigma$ of a difference equation of the form

$$
U(z, f) P(z, f)=Q(z, f),
$$

where $U(z, f), P(z, f)$ and $Q(z, f)$ are difference polynomials such that the total degree $\operatorname{deg}_{f} U(z, f)=n$ in $f(z)$ and its shifts, and $\operatorname{deg}_{f} Q(z, f) \leq n$. Moreover, we assume $U(z, f)$ contains just one term of maximal total degree in $f(z)$ and its shifts. Then for each $\varepsilon>0$,

$$
m(r, P(z, f))=O\left(r^{\sigma-1+\varepsilon}\right)+S(r, f),
$$

possibly outside of an exceptional set of finite logarithmic measure.

Lemma 2.4. (See [12, Corollary 2.5]) Let $f(z)$ be a meromorphic function of finite order $\sigma$ and let $\eta$ be a non-zero complex number. Then for each $\varepsilon>0$, we have

$$
m\left(r, \frac{f(z+\eta)}{f(z)}\right)+m\left(r, \frac{f(z)}{f(z+\eta)}\right)=O\left(r^{\sigma-1+\varepsilon}\right) .
$$


Lemma 2.5. (See [12, Theorem 2.1]) Let $f(z)$ be a meromorphic function with order $\sigma=\sigma(f), \sigma<+\infty$, and let $\eta$ be a fixed non-zero complex number, then for each $\varepsilon>0$, we have

$$
T(r, f(z+\eta))=T(r, f(z))+O\left(r^{\sigma-1+\varepsilon}\right)+O(\log r) .
$$

Lemma 2.6. (See [12, Theorem 2.2]) Let $f$ be a meromorphic function with exponent of convergence of poles $\lambda\left(\frac{1}{f}\right)=\lambda<\infty, \eta \neq$ 0 be fixed, then for each $\varepsilon>0$,

$$
N(r, f(z+\eta))=N(r, f(z))+O\left(r^{\lambda-1+\varepsilon}\right)+O(\log r) .
$$

\section{Proof of Theorems}

\section{Proof of Theorem 1.1}

(i) Firstly, we will prove $\lambda\left(\frac{1}{\frac{\Delta w}{w}}\right)=\sigma(w)$. By equation (1.2), Lemma 2.1, Lemma 2.5 and $|a|+|b| \neq 0$, we have

$$
\begin{aligned}
2 T(r, w(z)) & =T\left(r, \frac{a z+b+c w(z)}{w^{2}(z)}\right)+O(\log r) \\
& =T\left(r, \frac{w(z+1)+w(z-1)}{w(z)}\right)+O(\log r) \\
& \leq T\left(r, \frac{w(z+1)}{w(z)}\right)+T\left(r, \frac{w(z)}{w(z-1)}\right)+O(\log r) \\
& =2 T\left(r, \frac{w(z+1)}{w(z)}\right)+S\left(r, \frac{w(z+1)}{w(z)}\right)+O(\log r) \\
& \leq 2 T\left(r, \frac{w(z+1)}{w(z)}\right)+S(r, w) \\
& =2 T\left(r, \frac{\Delta w(z)}{w(z)}\right)+S(r, w),
\end{aligned}
$$

that is,

$$
T(r, w) \leq T\left(r, \frac{\Delta w(z)}{w(z)}\right)+S(r, w) .
$$

It follows from (3.1) and Lemma 2.4 that

$$
\begin{aligned}
N\left(r, \frac{\Delta w(z)}{w(z)}\right) & =T\left(r, \frac{\Delta w(z)}{w(z)}\right)-m\left(r, \frac{\Delta w(z)}{w(z)}\right) \\
& \geq T(r, w(z))+S(r, w) .
\end{aligned}
$$

Thus, $\lambda\left(\frac{1}{\frac{\Delta w}{w}}\right) \geq \sigma(w)$, that is $\lambda\left(\frac{1}{\frac{\Delta w}{w}}\right)=\sigma(w)$.

Next, we prove $\lambda\left(\frac{1}{\Delta w}\right)=\sigma(w)$. By equation (1.2),

$$
\begin{aligned}
\Delta w(z)-\Delta w(z-1) & =w(z+1)+w(z-1)-2 w(z) \\
& =\frac{a z+b}{w(z)}+c-2 w(z) \\
& =\frac{a z+b+c w(z)-2 w^{2}(z)}{w(z)} .
\end{aligned}
$$

From (3.2), Lemma 2.1, Lemma 2.5 and $|a|+|b| \neq 0$, we have

$$
\begin{aligned}
2 T(r, w(z)) & =T\left(r, \frac{a z+b+c w(z)-2 w^{2}(z)}{w(z)}\right)+O(\log r) \\
& =T(r, \Delta w(z)-\Delta w(z-1))+O(\log r) \\
& \leq T(r, \Delta w(z))+T(r, \Delta w(z-1))+O(\log r) \\
& =2 T(r, \Delta w(z))+S(r, \Delta w(z))+O(\log r) \\
& \leq 2 T(r, \Delta w(z))+S(r, w),
\end{aligned}
$$

that is,

$$
T(r, w(z)) \leq T(r, \Delta w(z))+S(r, w) .
$$

It follows from Lemma 2.5 that

$$
\begin{aligned}
T(r, \Delta w(z)) & \leq T(r, w(z+1))+T(r, w(z))+O(1) \\
& =2 T(r, w(z))+S(r, w)
\end{aligned}
$$

By equation (1.2), we obtain

$$
w(z)(w(z+1)+w(z-1))=a z+b+c w(z) .
$$

From (3.5) and Lemma 2.3, we see that for each $\varepsilon>0$, there is a subset $E_{1} \subset(1, \infty)$ having finite logarithmic measure such that for $|z|=r \notin[0,1] \cup E_{1}$,

$$
m(r, w(z+1)+w(z-1))=O\left(r^{\sigma(w)-1+\varepsilon}\right)+S(r, w) .
$$

It follows from equation (1.2), Lemma 2.1 and $|a|+|b| \neq 0$ that

$$
T(r, w(z+1)+w(z-1))=T\left(\frac{a z+b}{w(z)}+c\right)=T(r, w)+S(r, w) .
$$

From (3.6), (3.7) and Lemma 2.6, we obtain

$T(r, w(z))+S(r, w)=N(r, w(z+1)+w(z-1))$

$$
\leq N(r, w(z+1))+N(r, w(z-1))=2 N(r, w(z))+S(r, w) .
$$

It follows from Lemma 2.4 that

$$
m(r, \Delta w(z)) \leq m\left(r, \frac{\Delta w(z)}{w(z)}\right)+m(r, w(z))=m(r, w(z))+S(r, w) .
$$

From $(3.3)-(3.4)$ and $(3.8)-(3.9)$, we see

$$
\begin{aligned}
N(r, \Delta w(z)) \geq & T(r, \Delta w(z))-m(r, \Delta w(z)) \\
\geq & T(r, \Delta w(z))-(T(r, w(z)) \\
& \left.-\frac{1}{4} T(r, \Delta w(z))\right)+S(r, w) \\
= & \frac{5}{4} T(r, \Delta w(z))-T(r, w(z))+S(r, w) \\
\geq & \frac{1}{4} T(r, w(z))+S(r, w) .
\end{aligned}
$$

Thus, $\lambda\left(\frac{1}{\Delta w}\right) \geq \sigma(w)$, that is $\lambda\left(\frac{1}{\Delta w}\right)=\sigma(w)$.

(ii) For any $\eta \in \mathbb{C} \backslash\{0\}$, substituting $z+\eta$ into equation (1.2), we obtain

$$
w(z+\eta+1)+w(z+\eta-1)=\frac{a(z+\eta)+b}{w(z+\eta)}+c .
$$

Set $g(z)=w(z+\eta)$. Rewriting equation (3.10) as

$$
g(z)(g(z+1)+g(z-1))=c g(z)+(a(z+\eta)+b) .
$$

Denote

$$
P_{1}(z, g):=g(z)(g(z+1)+g(z-1))-c g(z)-(a(z+\eta)+b)=0 .
$$

Then, we have

$$
\begin{aligned}
P_{1}(z, z) & =z(z+1+z-1)-c z-(a(z+\eta)+b) \\
& =2 z^{2}-(a+c) z-(a \eta+b) \not \equiv 0 .
\end{aligned}
$$

From $P_{1}(z, z) \not \equiv 0$ and Lemma 2.2, we see

$$
m\left(r, \frac{1}{g(z)-z}\right)=S(r, g) .
$$

Thus, by Lemma 2.5, we have

$$
\begin{aligned}
N\left(r, \frac{1}{w(z+\eta)-z}\right) & =N\left(r, \frac{1}{g(z)-z}\right)=T(r, g)+S(r, g) \\
& =T(r, w(z+\eta))+S(r, w(z+\eta)) \\
& =T(r, w(z))+S(r, w) .
\end{aligned}
$$

Hence, for any $\eta \in \mathbb{C} \backslash\{0\}, \tau(w(z+\eta))=\sigma(w)$.

This completes the proof of Theorem 1.1.

Proof of Theorem 1.2

If $c=0$, equation (1.3) is a special case of equation (1.2). In what 
follows, we assume $c \neq 0$. (i) Firstly, we will prove $\lambda\left(\frac{1}{\frac{\Delta w}{w}}\right)=\sigma(w)$. By equation (1.3), Lemma 2.1, Lemma 2.5 and $c \neq 0$, we have

$$
\begin{aligned}
3 T(r, w(z)) & =T\left(r, \frac{(a z+b) w(z)+c}{w^{3}(z)}\right)+O(\log r) \\
& =T\left(r, \frac{w(z+1)+w(z-1)}{w(z)}\right)+O(\log r) \\
& \leq 2 T\left(r, \frac{\Delta w(z)}{w(z)}\right)+S(r, w),
\end{aligned}
$$

that is,

$$
\frac{3}{2} T(r, w(z)) \leq T\left(r, \frac{\Delta w(z)}{w(z)}\right)+S(r, w) .
$$

It follows from (3.11) and Lemma 2.4 that

$$
\begin{aligned}
N\left(r, \frac{\Delta w(z)}{w(z)}\right) & =T\left(r, \frac{\Delta w(z)}{w(z)}\right)-m\left(r, \frac{\Delta w(z)}{w(z)}\right) \\
& \geq \frac{3}{2} T(r, w(z))+S(r, w) .
\end{aligned}
$$

Thus, $\lambda\left(\frac{1}{\frac{\Delta w}{w}}\right) \geq \sigma(w)$, that is $\lambda\left(\frac{1}{\frac{\Delta w}{w}}\right)=\sigma(w)$.

Next, we prove $\lambda\left(\frac{1}{\Delta w}\right)=\sigma(w)$. By equation (1.3),

$$
\Delta w(z)-\Delta w(z-1)=\frac{c+(a z+b) w(z)-2 w^{3}(z)}{w^{2}(z)} .
$$

From (3.12), Lemma 2.1, Lemma 2.5 and $c \neq 0$, we have

$$
\begin{aligned}
3 T(r, w(z)) & =T\left(r, \frac{c+(a z+b) w(z)-2 w^{3}(z)}{w^{2}(z)}\right)+O(\log r) \\
& =T(r, \Delta w(z)-\Delta w(z-1))+O(\log r) \\
& \leq 2 T(r, \Delta w(z))+S(r, w),
\end{aligned}
$$

that is,

$$
\frac{3}{2} T(r, w(z)) \leq T(r, \Delta w(z))+S(r, w) .
$$

By Lemma 2.4,

$$
m(r, \Delta w(z))=m\left(r, \frac{\Delta w(z)}{w(z)}\right)+m(r, w(z)) \leq T(r, w(z))+S(r, w) .
$$

It follows from (3.13) and (3.14) that

$$
\begin{aligned}
N(r, \Delta w(z)) & =T(r, \Delta w(z))-m(r, \Delta w(z)) \\
& \geq \frac{3}{2} T(r, w(z))-T(r, w(z))+S(r, w) \\
& =\frac{1}{2} T(r, w(z))+S(r, w) .
\end{aligned}
$$

Thus, $\lambda\left(\frac{1}{\Delta w}\right) \geq \sigma(w)$, that is $\lambda\left(\frac{1}{\Delta w}\right)=\sigma(w)$.

(ii) For any $\eta \in \mathbb{C} \backslash\{0\}$, substituting $z+\eta$ into equation (1.3), we obtain

$$
w(z+\eta+1)+w(z+\eta-1)=\frac{a(z+\eta)+b}{w(z+\eta)}+\frac{c}{w(z+\eta)^{2}} .
$$

Set $g(z)=w(z+\eta)$. Then (3.15) can be rewritten as

$$
g^{2}(z)(g(z+1)+g(z-1))=g(z)(a(z+\eta)+b)+c .
$$

Denote

$P_{2}(z, g):=g^{2}(z)(g(z+1)+g(z-1))-g(z)(a(z+\eta)+b)-c=0$.

Then, we have

$$
P_{2}(z, z)=z^{2}(z+1+z-1)-z(a(z+\eta)+b)-c \not \equiv 0 .
$$

From $P_{2}(z, z) \not \equiv 0$ and Lemma 2.2, we see

$$
m\left(r, \frac{1}{g(z)-z}\right)=S(r, g) .
$$

Thus, by Lemma 2.5 , we have

$$
\begin{aligned}
N\left(r, \frac{1}{w(z+\eta)-z}\right) & =N\left(r, \frac{1}{g(z)-z}\right)=T(r, g)+S(r, g) \\
& =T(r, w(z+\eta))+S(r, w(z+\eta)) \\
& =T(r, w)+S(r, w) .
\end{aligned}
$$

Hence, for any $\eta \in \mathbb{C} \backslash\{0\}, \tau(w(z+\eta))=\sigma(w)$.

This completes the proof of Theorem 1.2.

Proof of Theorem 1.3

Using the same method as the proof of Theorem 1.1, we can easily obtain $\lambda\left(\frac{1}{\frac{\Delta w}{w}}\right)=\lambda(\Delta w)=\sigma(w)$.

\section{Proof of Theorem 1.4}

(i) In what follows, we consider three cases: Case 1, $c=0$; Case $2, c \neq 0$, either $a=0, b-c=0$, or $a=0, b+c=0$; Case $3, c \neq 0$, either $a \neq 0$, or $b-c \neq 0$, or $b+c \neq 0$.

Case $1, c=0$. Firstly, we prove we prove $\lambda\left(\frac{1}{\frac{\Delta w}{w}}\right)=\sigma(w)$. By equation (1.5), Lemma 2.1, Lemma 2.5 and $|a|+|b| \neq 0$, we have

$$
\begin{aligned}
2 T(r, w(z)) & =T\left(r, \frac{a z+b}{1-w^{2}(z)}\right)+O(\log r) \\
& =T\left(r, \frac{w(z+1)+w(z-1)}{w(z)}\right)+O(\log r) \\
& \leq 2 T\left(r, \frac{\Delta w(z)}{w(z)}\right)+S(r, w),
\end{aligned}
$$

that is,

$$
T(r, w(z)) \leq T\left(r, \frac{\Delta w(z)}{w(z)}\right)+S(r, w) .
$$

It follows from (3.16) and Lemma 2.4 that

$$
\begin{aligned}
N\left(r, \frac{\Delta w(z)}{w(z)}\right) & =T\left(r, \frac{\Delta w(z)}{w(z)}\right)-m\left(r, \frac{\Delta w(z)}{w(z)}\right) \\
& \geq T(r, w(z))+S(r, w) .
\end{aligned}
$$

Thus, $\lambda\left(\frac{1}{\frac{\Delta w}{w}}\right) \geq \sigma(w)$, that is $\lambda\left(\frac{1}{\frac{\Delta w}{w}}\right)=\sigma(w)$.

Next, we prove $\lambda\left(\frac{1}{\Delta w}\right)=\sigma(w)$. By equation (1.5),

$$
\Delta w(z)-\Delta w(z-1)=\frac{2 w^{3}(z)+(a z+b-2) w(z)}{1-w^{2}(z)} .
$$

From (3.17), Lemma 2.1, Lemma 2.5 and $|a|+|b| \neq 0$, we have

$$
\begin{aligned}
3 T(r, w(z)) & =T\left(r, \frac{2 w^{3}(z)+(a z+b-2) w(z)}{1-w^{2}(z)}\right)+O(\log r) \\
& =T(r, \Delta w(z)-\Delta w(z-1))+O(\log r) \\
& \leq 2 T(r, \Delta w(z))+S(r, w),
\end{aligned}
$$

that is,

$$
\frac{3}{2} T(r, w(z)) \leq T(r, \Delta w(z))+S(r, w) .
$$

From (3.14) and (3.18), we have

$$
\begin{aligned}
N(r, \Delta w(z)) & =T(r, \Delta w(z))-m(r, \Delta w) \\
& \geq \frac{3}{2} T(r, w(z))-T(r, w(z))+S(r, w) \\
& =\frac{1}{2} T(r, w(z))+S(r, w) .
\end{aligned}
$$

Thus, $\lambda\left(\frac{1}{\Delta w}\right) \geq \sigma(w)$, that is $\lambda\left(\frac{1}{\Delta w}\right)=\sigma(w)$.

Case 2, $c \neq 0$, either $a=0, b-c=0$, or $a=0, b+c=0$. We divide 
this proof into the following two subcases.

Case 2.1, $c \neq 0, a=0, b-c=0$. Firstly, we prove $\lambda\left(\frac{1}{\frac{\Delta w}{w}}\right)=\sigma(w)$. By equation (1.5), Lemma 2.1, Lemma 2.5 and $b=c(\neq 0)$, we have

$$
\begin{aligned}
2 T(r, w(z)) & =T\left(r, \frac{b}{w(z)(1-w(z))}\right)+O(1) \\
& =T\left(r, \frac{w(z+1)+w(z-1)}{w(z)}\right)+O(1) \\
& \leq 2 T\left(r, \frac{\Delta w(z)}{w(z)}\right)+S(r, w),
\end{aligned}
$$

hence,

$$
T(r, w(z)) \leq T\left(r, \frac{\Delta w(z)}{w(z)}\right)+S(r, w) .
$$

From (3.19) and Lemma 2.4, we see

$$
\begin{aligned}
N\left(r, \frac{\Delta w(z)}{w(z)}\right) & =T\left(r, \frac{\Delta w(z)}{w(z)}\right)-m\left(r, \frac{\Delta w(z)}{w(z)}\right) \\
& \geq T(r, w(z))+S(r, w) .
\end{aligned}
$$

Thus, $\lambda\left(\frac{1}{\frac{\Delta w}{w}}\right) \geq \sigma(w)$, that is $\lambda\left(\frac{1}{\frac{\Delta w}{w}}\right)=\sigma(w)$.

Next, we prove $\lambda\left(\frac{1}{\Delta w}\right)=\sigma(w)$. By equation (1.5),

$$
\Delta w(z)-\Delta w(z-1)=\frac{2 w^{2}(z)-2 w(z)+b}{1-w(z)} .
$$

From (3.20), Lemma 2.1, Lemma 2.5 and $b=c(\neq 0)$, we have

$$
\begin{aligned}
2 T(r, w(z)) & =T\left(r, \frac{2 w^{2}(z)-2 w(z)+b}{1-w(z)}\right)+O(1) \\
& =T(r, \Delta w(z)-\Delta w(z-1))+O(1) \\
& \leq 2 T(r, \Delta w(z))+S(r, w)
\end{aligned}
$$

that is,

$$
T(r, w(z)) \leq T(r, \Delta w(z))+S(r, w) .
$$

By equation (1.5), we obtain

$$
w(z)(w(z+1)+w(z-1))=w(z+1)+w(z-1)-b .
$$

From (3.22) and Lemma 2.3, we see that for each $\varepsilon>0$, there is a subset $E_{2} \subset(1, \infty)$ having finite logarithmic measure such that for $|z|=r \notin[0,1] \cup E_{2}$,

$$
m(r, w(z+1)+w(z-1))=O\left(r^{\sigma(w)-1+\varepsilon}\right)+S(r, w) .
$$

By equation (1.5), Lemma 2.1 and $b=c(\neq 0)$, we see

$$
T(r, w(z+1)+w(z-1))=T\left(\frac{b}{1-w(z)}\right)=T(r, w)+S(r, w) .
$$

From (3.23), (3.24) and Lemma 2.6, we obtain

$$
\begin{aligned}
T(r, w(z))+S(r, w) & =N(r, w(z+1)+w(z-1)) \\
& \leq 2 N(r, w(z))+S(r, w)
\end{aligned}
$$

From (3.4), (3.9), (3.21) and (3.25), we see

$$
\begin{aligned}
N(r, \Delta w(z))= & T(r, \Delta w(z))-m(r, \Delta w(z)) \\
\geq & T(r, \Delta w(z))-(T(r, w(z)) \\
& \left.-\frac{1}{4} T(r, \Delta w(z))\right)+S(r, w) \\
= & \frac{5}{4} T(r, \Delta w(z))-T(r, w(z))+S(r, w) \\
\geq & \frac{1}{4} T(r, w(z))+S(r, w) .
\end{aligned}
$$

Thus, $\lambda\left(\frac{1}{\Delta w}\right) \geq \sigma(w)$, that is $\lambda\left(\frac{1}{\Delta w}\right)=\sigma(w)$.

Case 2.2, $c \neq 0, a=0, b+c=0$. Using the same method as the proof of subcase 2.1, we can also obtain $\lambda\left(\frac{1}{\Delta w}\right)=\lambda\left(\frac{1}{\frac{\Delta w}{w}}\right)=\sigma(w)$; Case 3, $c \neq 0$, either $a \neq 0$, or $b-c \neq 0$, or $b+c \neq 0$. Firstly, we prove $\lambda\left(\frac{1}{\frac{\Delta w}{w}}\right)=\sigma(w)$. By equation (1.5), Lemma 2.1, Lemma 2.5 and $c \neq 0$, either $a \neq 0$, or $b-c \neq 0$, or $b+c \neq 0$, we have

$$
\begin{aligned}
3 T(r, w(z)) & =T\left(r, \frac{(a z+b) w(z)+c}{w(z)\left(1-w^{2}(z)\right)}\right)+O(\log r) \\
& =T\left(r, \frac{w(z+1)+w(z-1)}{w(z)}\right)+O(\log r) \\
& \leq 2 T\left(r, \frac{\Delta w(z)}{w(z)}\right)+S(r, w),
\end{aligned}
$$

that is,

$$
\frac{3}{2} T(r, w(z)) \leq T\left(r, \frac{\Delta w(z)}{w(z)}\right)+S(r, w)
$$

From (3.26) and Lemma 2.4, we see

$$
\begin{aligned}
N\left(r, \frac{\Delta w(z)}{w(z)}\right) & =T\left(r, \frac{\Delta w(z)}{w(z)}\right)-m\left(r, \frac{\Delta w(z)}{w(z)}\right) \\
& \geq \frac{3}{2} T(r, w(z))+S(r, w) .
\end{aligned}
$$

Thus, $\lambda\left(\frac{1}{\frac{\Delta w}{w}}\right) \geq \sigma(w)$, that is $\lambda\left(\frac{1}{\frac{\Delta w}{w}}\right)=\sigma(w)$.

Next, we prove $\lambda\left(\frac{1}{\Delta w}\right)=\sigma(w)$. By equation (1.5),

$$
\Delta w(z)-\Delta w(z-1)=\frac{2 w^{3}(z)+(a z+b-2) w(z)+c}{1-w^{2}(z)} .
$$

From (3.27), Lemma 2.1, Lemma 2.5 and $c \neq 0$, either $a \neq 0$, or $b-c \neq 0$, or $b+c \neq 0$, we have

$$
\begin{aligned}
3 T(r, w) & =T\left(r, \frac{2 w^{3}(z)+(a z+b-2) w(z)+c}{1-w^{2}(z)}\right)+O(\log r) \\
& =T(r, \Delta w(z)-\Delta w(z-1))+O(\log r) \\
& \leq 2 T(r, \Delta w(z))+S(r, w),
\end{aligned}
$$

that is,

$$
\frac{3}{2} T(r, w(z)) \leq T(r, \Delta w(z))+S(r, w) .
$$

From (3.14) and (3.28), we have

$$
\begin{aligned}
N(r, \Delta w(z)) & =T(r, \Delta w(z))-m(r, \Delta w(z)) \\
& \geq \frac{3}{2} T(r, w(z))-T(r, w(z))+S(r, w) \\
& =\frac{1}{2} T(r, w(z))+S(r, w) .
\end{aligned}
$$

Thus, $\lambda\left(\frac{1}{\Delta w}\right) \geq \sigma(w)$, that is $\lambda\left(\frac{1}{\Delta w}\right)=\sigma(w)$.

(ii) For any $\eta \in \mathbb{C} \backslash\{0\}$, substituting $z+\eta$ into equation (1.5), we obtain

$$
w(z+\eta+1)+w(z+\eta-1)=\frac{(a(z+\eta)+b) w(z+\eta)+c}{1-w(z+\eta)^{2}},
$$

Set $g(z)=w(z+\eta)$. Then (3.29) can be rewritten as

$$
\left(1-g^{2}(z)\right)(g(z+1)+g(z-1))=g(z)(a(z+\eta)+b)+c .
$$

Denote

$$
\begin{aligned}
P_{3}(z, g):= & \left(1-g^{2}(z)\right)(g(z+1)+g(z-1)) \\
& -g(z)(a(z+\eta)+b)-c=0 .
\end{aligned}
$$

Then, we have

$$
P_{3}(z, z)=\left(1-z^{2}\right)(z+1+z-1)-z(a(z+\eta)+b)-c \not \equiv 0 .
$$


From $P_{3}(z, z) \not \equiv 0$ and Lemma 2.2, we see that

$$
m\left(r, \frac{1}{g(z)-z}\right)=S(r, g) .
$$

Thus, by Lemma 2.5, we have

$$
\begin{aligned}
N\left(r, \frac{1}{w(z+\eta)-z}\right) & =N\left(r, \frac{1}{g(z)-z}\right)=T(r, g)+S(r, g) \\
& =T(r, w(z+\eta))+S(r, w(z+\eta)) \\
& =T(r, w(z))+S(r, w) .
\end{aligned}
$$

Hence, for any $\eta \in \mathbb{C} \backslash\{0\}, \tau(w(z+\eta))=\sigma(w)$.

This completes the proof of Theorem 1.4.

\section{Acknowledgement}

The authors would like to thank the referee for his/her thorough reviewing with constructive suggestions and comments to the paper.

\section{References}

[1] A.Z. Mokhon'ko, "On the Nevanlinna characteristics of some meromorphic functions, in 'Theory of functions, functional analysis and their applicatins'”, Izd-vo Khar'kovsk. Un-ta, 14(1971), 83-87.

[2] I. Laine, Nevanlinna Theory and Complex Differential Equations, W. de Gruyter, Berlin, 1993.

[3] I. Laine, C.C. Yang, "Clunie theorems for difference and q-difference polynomials", J. Lond. Math. Soc, 76(2007), 556-566.

[4] J. Heittokangas, R. Korhonen, I. Laine, J. Rieppo, J. Zhang, "Value sharing results for shifts of meromorphic functions and sufficient conditions for periodicity", J. Math. Anal. Appl, 355(2009), 352-363.

[5] J. Heittokangas, R. Korhonen, I. Laine, J. Rieppo, K. Hohge, "Complex difference equations of Malmquist type", Comput. Methods. Funct. Theory, 1(2001), 27-39.

[6] K. Ishizaki, N. Yanagihara, "Wiman-Valiron method for difference equations", Nagoya Math. J, 175(2004), 75-102.

[7] M.R. Chen, Z.X. Chen, "On properties of finite order meromorphic solutions of a certain difference Painlevé I equation", Bull. Aust. Math. Soc, 85(2012), 463-475.

[8] R.G. Halburd, R. Korhonen, "Difference analogue of the lemma on the logarithmic derivative with applications to difference equations", $J$ Math. Anal. Appl, 314(2006), 477-487.

[9] R.G. Halburd, R. Korhonen, "Finite-order meromorphic solutions and the discrete Painlevé equations", Proc. Lond. Math. Soc, 94(2007),

[10] W.K. Hayman, Meromorphic Function, Clarendon Press, Oxford, 1964

[11] Y.M. Chiang, S.J. Feng, "On the growth of logarithmic differences, difference quotients and logarthmic derivatives of meromorphic functions", Trans. Amer. Math. Soc, 361(2009), 3767-3791.

[12] Y.M. Chiang, S.J. Feng, "On the Nevanlinna characteristic of $f(z+$ $\eta$ ) and difference equations in the complex plane", Ramanujan $J$, 16(2008), 105-129.

[13] Z.X. Chen, K.H. Shon, "Value distribution of meromorphic solutions of certain difference Painlevé equations", J. Math. Anal. Appl, 364(2010), 556-566.

[14] Z.X. Chen, "On properties of meromorphic solutions for some difference equations", Kodai Math. J, 34(2011), 244-256. 